\title{
Smac mimetic-induced upregulation of interferon- $\beta$ sensitizes glioblastoma to temozolomide-induced cell death
}

\author{
V Marschall ${ }^{1}$ and S Fulda ${ }^{\star, 1,2,3}$
}

\begin{abstract}
Inhibitor of apoptosis (IAP) proteins are frequently expressed at high levels in cancer cells and represent attractive therapeutic targets. We previously reported that the Smac (second mitochondria-derived activator of caspases) mimetic BV6, which antagonizes IAP proteins, sensitizes glioblastoma cells to temozolomide (TMZ)-induced cell death in a nuclear factor- $\kappa B$ (NF- $\kappa$ B)dependent manner. However, BV6-induced NF- $\kappa$ B target genes responsible for this synergistic interaction have remained elusive. Using whole-genome gene expression profiling, we here identify BV6-stimulated, NF- $\kappa \mathrm{B}$-dependent transcriptional upregulation of interferon- $\beta$ (IFN $\beta$ ) and IFN-mediated proapoptotic signaling as critical events that mediate BV6/TMZ-induced apoptosis. Knockdown of IFN $\beta$ significantly rescues cells from BV6/TMZ-induced cell death. Similarly, silencing of the corresponding receptor IFN $\alpha / \beta$ receptor (IFNAR) confers a significant protection against apoptosis, demonstrating that IFN $\beta$ and IFN signaling are required for BV6/TMZ-mediated cell death. Moreover, BV6 and TMZ cooperate to transcriptionally upregulate the proapoptotic B-cell lymphoma 2 family proteins Bax (Bcl-2-associated X protein) or Puma (p53-upregulated modulator of apoptosis). Knockdown of Bax or Puma significantly decreases BV6/TMZ-induced apoptosis, showing that both proteins are necessary for apoptosis. By identifying IFN $\beta$ as a key mediator of BV6/TMZ-induced apoptosis, our study provides novel insights into the underlying molecular mechanisms of Smac mimetic-mediated chemosensitization with important implications for the development of novel treatment strategies for glioblastoma.
\end{abstract}

Cell Death and Disease (2015) 6, e1888; doi:10.1038/cddis.2015.235; published online 17 September 2015

Glioblastoma is the most common primary malignant brain tumor and current treatment options include surgical resection, radiation and chemotherapy with the alkylating agent temozolomide (TMZ). ${ }^{1}$ However, despite aggressive treatment regimens, the prognosis of patients suffering from glioblastoma is still very poor, ${ }^{2}$ highlighting the high medical need for novel treatment strategies.

Evasion of programmed cell death is one of the hallmarks of human cancers ${ }^{3}$ and promotes tumorigenesis as well as treatment resistance. ${ }^{4}$ Apoptosis is a common form of programmed cell death that can be engaged via the intrinsic (mitochondrial) or extrinsic (death receptor) pathway. ${ }^{5}$ Activation of the intrinsic pathway is controlled by pro- and antiapoptotic B-cell lymphoma 2 (Bcl-2) family protein, including Bcl-2 family proteins such as p53-upregulated modulator of apoptosis (Puma) or Bcl-2-associated $\mathrm{X}$ protein (Bax). Following engagement of the mitochondrial pathway, mitochondrial intermembrane space proteins are released into the cytosol, including second mitochondria-derived activator of caspases (Smac). ${ }^{6}$ Smac binds to and neutralizes Inhibitor of Apoptosis (IAP) proteins, a family of antiapoptotic proteins, thereby promoting activation of caspases and apoptosis. ${ }^{7}$ In addition, binding of Smac to IAP proteins that harbor a Really Interesting New Gene (RING) domain with E3 ligase activity triggers their autoubiquitination and proteasomal degradation, which in turn leads to stabilization of nuclear factor- $\kappa \mathrm{B}(\mathrm{NF}-\kappa \mathrm{B})$-inducing kinase $(\mathrm{NIK})$ and activation of noncanonical NF- $k$ B signaling. ${ }^{8,9}$

IAP proteins are expressed at high levels in various cancers and represent attractive targets for therapeutic intervention. ${ }^{7}$ BV6 is a synthetically designed Smac mimetic that mimics the N-terminal part of endogenous Smac protein. ${ }^{8} \mathrm{We}$ previously reported that Smac mimetics such as BV6 sensitize glioblastoma cells to chemotherapy- or $\gamma$-irradiation-induced apoptosis in an NF-kB-dependent manner. ${ }^{10-12}$ Although BV6-stimulated NF- $K \mathrm{~B}$ activation was demonstrated to be critically required for Smac mimetic-mediated sensitization of glioblastoma cells towards $\mathrm{TMZ}$, the proapoptotic NF-KBregulated target genes that mediate this chemosensitization have so far remained elusive. ${ }^{12}$ While tumor necrosis factor $a$ (TNFa), a prototypic NF- $\kappa$ B target gene, was shown to mediate apoptosis via an autocrine/paracrine loop upon treatment with

\footnotetext{
${ }^{1}$ Institute for Experimental Cancer Research in Pediatrics, Goethe-University, Komturstrasse 3a, Frankfurt 60528, Germany; ${ }^{2}$ German Cancer Consortium (DKTK), Heidelberg, Germany and ${ }^{3}$ German Cancer Research Center (DKFZ), Heidelberg, Germany

${ }^{*}$ Corresponding author: S Fulda, Institute for Experimental Cancer Research in Pediatrics, Goethe-University, Komturstrasse 3a, Frankfurt 60528, Germany. Tel: +49 69 67866557; Fax: +49 69 6786659157; E-mail: simone.fulda@kgu.de

Abbreviations: Bak, Bcl-2 homologous antagonist/killer; Bax, Bcl-2-associated X protein; Bcl-2, B-cell lymphoma 2; Bid, BH3-interacting domain death agonist; Bim, Bcl2-interacting mediator of cell death; Bmf, Bcl-2-modifying factor; IAP, inhibitor of apoptosis; IFN, interferon; IFNAR, IFN $\alpha / \beta$ receptor; I $\kappa_{\mathrm{B}} \alpha$-SR, IKB $\alpha$ superrepressor IRF, interferon regulatory factor; ISG, IFN-stimulated gene; NF- $\kappa \mathrm{B}$, nuclear factor- $\kappa \mathrm{B}$; NIK, nuclear factor- $\kappa \mathrm{B}$-inducing kinase; Noxa/PMAIP1, phorbol-12-myristate-13acetate-induced protein 1; Puma, p53-upregulated modulator of apoptosis; RING, Really Interesting New Gene; Smac, second mitochondria-derived activator of caspases; TMZ, temozolomide; TNF $\alpha$, tumor necrosis factor- $\alpha$; TNFR1, tumor necrosis factor- $\alpha$ receptor 1

Received 05.5.2015; revised 09.7.2015; accepted 20.7.2015; Edited by G Dewson
} 
Smac mimetic alone $e^{8,9,13}$ or in combination with anticancer drugs in different carcinoma cell lines, ${ }^{14}$ TNF $a$ was found to be largely dispensable for BV6/TMZ-induced apoptosis in glioblastoma cells. ${ }^{12}$ In the present study, we therefore aimed at discovering novel NF- $k \mathrm{~B}$-dependent factors that are required for the cooperative anticancer activity of BV6 and TMZ, the prototypic chemotherapeutic agent used for the treatment of glioblastoma.

\section{Results}

BV6/TMZ cotreatment upregulates IFN-responsive genes before cell death induction. Initially, we assessed cell death upon treatment with the chemotherapeutic agent $\mathrm{TMZ}$ and the Smac mimetic BV6 using the glioblastoma cell lines A172 and T98G to confirm that Smac mimetic enhances TMZ-induced apoptosis, as we reported previously. ${ }^{12}$ Indeed, determination of DNA fragmentation as a marker of apoptosis demonstrated that BV6 sensitizes glioblastoma cells to TMZinduced apoptotic cell death (Figure 1a and Supplementary Figure 1). As Smac mimetics have been described to activate NF- $K \mathrm{~B}$ signaling, we stably overexpressed dominant-negative
I $\mathrm{B} a$-superrepressor ( $\left.\left.\right|_{\kappa} \mathrm{B} a-\mathrm{SR}\right)$ to block NF-kB signaling ${ }^{15}$ (Figure 1c). Remarkably, inhibition of $\mathrm{NF}-\kappa \mathrm{B}$ by $I_{k} \mathrm{~B} a-\mathrm{SR}$ almost completely rescued cells from BV6/TMZ-induced apoptosis (Figure $1 \mathrm{~b}$ ), underlining the proapoptotic role of NF- $\kappa \mathrm{B}$ signaling in this context. To further investigate which BV6-induced NF- $k$ B target genes are responsible for proapoptotic signaling upon BV6/TMZ cotreatment, we performed whole-genome expression profiling using an expression bead chip hybridization assay. Whole-genome expression data were ranked according to fold upregulation comparing A172 glioblastoma cells expressing empty vector (EV) with and without BV6/TMZ treatment. Expression data showing upregulation in A172 glioblastoma cells expressing $I_{k} \mathrm{~B} a-\mathrm{SR}$ served as control to identify background expression of nonNF- $\kappa$ B-regulated genes. BV6-treated cells showed a similar expression pattern as BV6/TMZ cotreated cells (data not shown). Interestingly, gene set enrichment analysis (GSEA) revealed upregulation of interferon (IFN)-responsive genes after BV6/TMZ treatment (Table 1 and Supplementary Figure 2). These results demonstrate that $\mathrm{BV} 6 / \mathrm{TMZ}$ treatment upregulates IFN-responsive genes in an NF- $\kappa$ B-dependent manner.
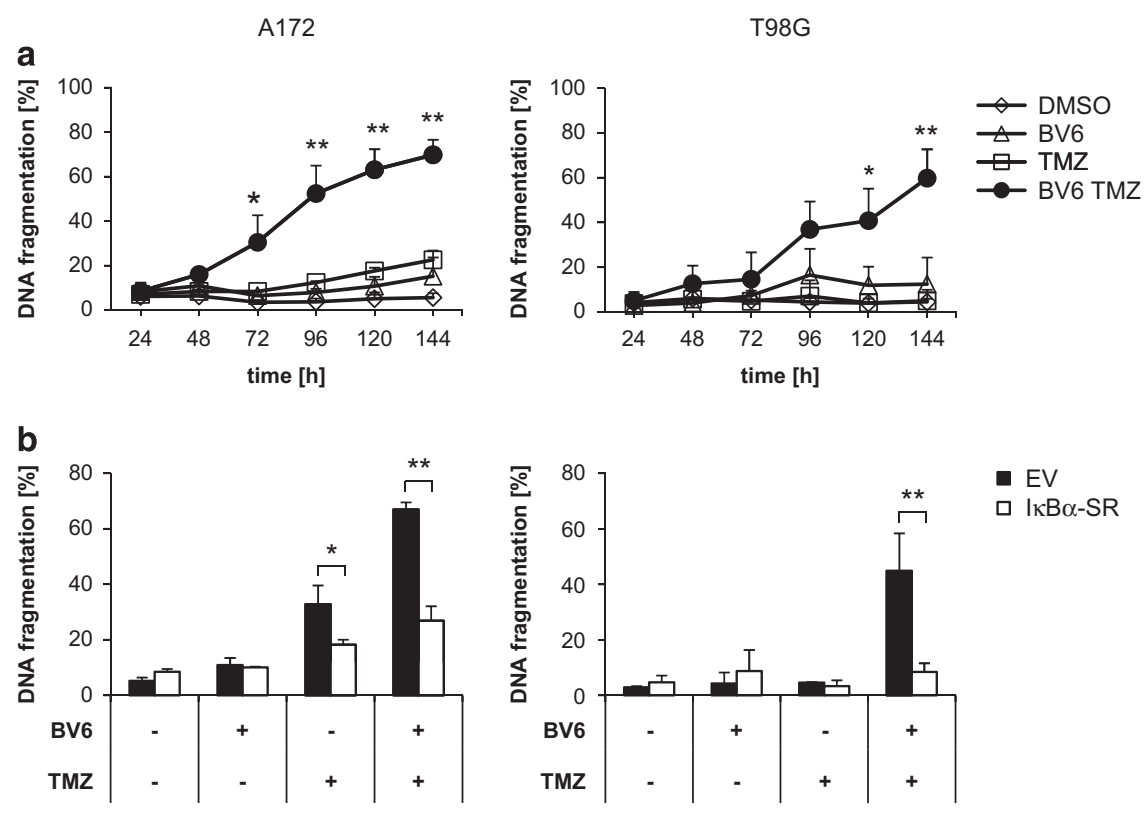

- EV

C
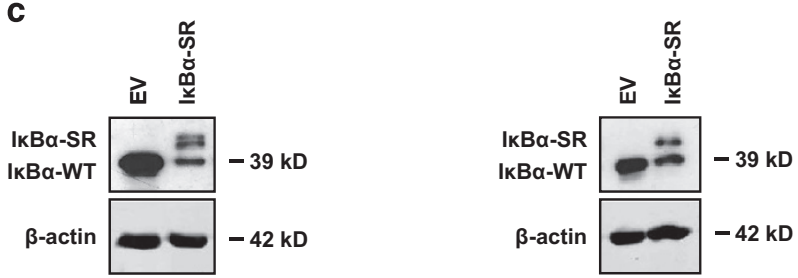

Figure 1 BV6/TMZ cotreatment upregulates IFN-responsive genes. (a) A172 cells (left) or T98G cells (right) were treated for indicated times with $100 \mu \mathrm{M}$ TMZ and/or $2 \mu \mathrm{M}$ BV6 (A172) or $4 \mu \mathrm{M}$ BV6 (T98G) or dimethyl sulfoxide (DMSO). Apoptosis was determined by fluorescence-activated cell sorting (FACS) analysis of DNA fragmentation of PIstained nuclei. Mean values +S.D. of three to four independent experiments performed in triplicate are shown; ${ }^{*} P<0.05 ;{ }^{* \star} P<0.01$ compared with all other settings. (b) A172 cells (left) or T98G cells (right) stably expressing $\mathrm{I}_{\mathrm{B}} \alpha$-SR or EV were treated for $120 \mathrm{~h}$ with $100 \mu \mathrm{M}$ TMZ and/or $2 \mu \mathrm{M}$ BV6 (A172) or $4 \mu \mathrm{M}$ BV6 (T98G) or DMSO. Apoptosis was determined by FACS analysis of DNA fragmentation of PI-stained nuclei. Mean values $+S$.D. of three independent experiments performed in triplicate are shown; ${ }^{*} P<0.05$;

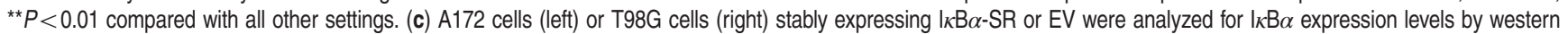
blotting. Expression of $\beta$-actin served as a loading control. A representative experiment of two independent experiments is shown 
Table 1 BV6/TMZ treatment upregulates ISGs

\begin{tabular}{lc}
\hline Enriched gene set & ES \\
\hline MOSERLE_IFNA_RESPONSE & 0.82 \\
SANA_RESPONSE_TO_IFNG_UP & 0.81 \\
BROWNE_INTERFERON_RESPONSIVE_GENES & 0.77 \\
DER_IFN_GAMMA_RESPONSE_UP & 0.76 \\
REACTOME_INTERFERON_GAMMA_SIGNALING & 0.74 \\
REACTOME_RIG_I_MDA5_MEDIATED_INDUCTION_OF_- & 0.73 \\
IFN_ALPHA_BETA_PATHWAYS & \\
BOSCO_INTERFERON_INDUCED_ANTIVIRAL_MODULE & 0.72 \\
HECKER_IFNB1_TARGETS & 0.71 \\
REACTOME_INTERFERON_ALPHA_BETA_SIGNALING & 0.70 \\
DER_IFN_ALPHA_RESPONSE_UP & 0.69 \\
DER_IFN_BETA_RESPONSE_UP & 0.65 \\
REACTOME_INTERFERON_SIGNALING & 0.63 \\
& \\
\hline
\end{tabular}

A172 cells stably expressing $\mathrm{I} \mathrm{B} a$-SR or $\mathrm{EV}$ were treated for $9 \mathrm{~h}$ with $100 \mu \mathrm{M}$ TMZ and/or $2 \mu \mathrm{M}$ BV6 or DMSO. Whole-genome expression profiling was performed. Genes with similar regulation in A172 cells expressing IkB $a-S R$ served as control for background expression of non-NF-kB-stimulated genes. GSEA was performed comparing TMZ/BV6-treated cells to all other settings. The enrichment score (ES) of IFN signaling-mediated gene sets out of the top 100 regulated gene sets upon BV6/TMZ treatment are shown. The false discovery rate for all gene sets shown in the table is $<0.0$. Mean values of three independent experiments are shown

BV6-mediated upregulation of IFN $\beta$ sensitizes glioblastoma cells to TMZ-induced apoptosis. Next, we investigated whether IFNs are involved in BV6/TMZ-induced cell death. Type I IFNs such as IFN $a$ and IFN $\beta$ have been reported to synergize with $T M Z$ in cell death induction in glioblastoma cells. ${ }^{16,17}$ Therefore, we analyzed mRNA expression levels of IFN $a$ and IFN $\beta$ upon treatment with BV6 and/or TMZ using quantitative real-time-PCR (qRT-PCR) analysis, as IFNs were not represented on the expression bead chip hybridization assay. Interestingly, IFN $\beta$ was upregulated upon BV6 single treatment, as well as upon BV6/TMZ cotreatment (Figure 2a). In addition, BV6stimulated transcriptional upregulation of IFN $\beta$ was inhibited in I $\kappa \mathrm{B} a$-SR-overexpressing cells (Figure $2 \mathrm{a}$ ), demonstrating that it occurs in an NF- $k B$-dependent manner. In contrast to IFN $\beta$, IFNa mRNA expression levels remained largely unchanged upon treatment with BV6 and/or TMZ (Figure 2b).

To explore whether IFN $\beta$ acts in concert with TMZ to cause cell death, we treated glioblastoma cells with IFN $\beta$ alone and in combination with TMZ. Intriguingly, IFN $\beta$ significantly increased TMZ-induced cell death in A172 and T98G cells compared to treatment with either agent alone (Figure 2c). In addition to IFN $\beta$, IFN $a$ significantly enhanced TMZ-induced cell death in glioblastoma cells (Supplementary Figure 3). This set of experiments demonstrates that BV6/TMZ induces upregulation of IFN $\beta$ in an NF- $k B$-dependent manner and that IFN $\beta$ and TMZ cooperate to induce apoptosis in glioblastoma cells.

IFN $\beta$ is required for $B V 6 / T M Z$-induced apoptosis. To examine whether IFN $\beta$ is required for BV6/TMZ-induced cell death, we created IFN $\beta$-knockdown cells (Figure 3a). Remarkably, silencing of IFN $\beta$ significantly inhibited BV6/TMZ-mediated cell death (Figure 3b). Type I IFNs such as IFN $a$ and IFN $\beta$ bind to a transmembrane receptor termed IFN $\alpha / \beta$ receptor (IFNAR) and thereby induce transcriptional activation of IFN-stimulated genes (ISGs). ${ }^{18}$ To examine whether IFNAR signaling is involved in BV6/TMZ-induced cell death, we generated IFNAR1-knockdown cells (Figure 3c). Silencing of IFNAR1 significantly reduced BV6/TMZ-mediated cell death (Figure 3d). In addition, knockdown of IFNAR1 rescued cells from IFN $\beta / T M Z$-induced cell death (Figure $3 e$ ). Taken together, this set of experiments demonstrates that IFN $\beta$ has an important role in mediating BV6/TMZ-induced cell death.

BV6/TMZ-induced apoptosis is mediated by cooperative upregulation of Puma and Bax. ISGs have been described to mediate IFN-induced apoptosis via upregulation of proapoptotic proteins, including proteins of the mitochondrialdependent cell death pathway. ${ }^{19}$ To link BV6/TMZ-mediated activation of IFN signaling to activation of the mitochondrial apoptotic pathway, we analyzed the expression levels of various proapoptotic Bcl-2 family members (Supplementary Figure 4a). BV6/TMZ cotreatment significantly upregulated mRNA levels of Puma and Bax, whereas no consistent upregulation was observed for Bak (Bcl-2 homologous antagonist/killer), Noxa, Bim (Bcl-2-interacting mediator of cell death), Bid (BH3-interacting domain death agonist) and Bmf (Bcl-2-modifying factor; Figures $4 \mathrm{a}$ and $\mathrm{b}$ and Supplementary Figure 4a). Also, Puma and Bax were upregulated on the protein level upon BV6/TMZ treatment (Supplementary Figure 4b). To determine whether Puma and Bax are required of BV6/TMZ-induced cell death, we created Puma- or Bax-knockdown cells (Figures $4 \mathrm{c}$ and e). Interestingly, knockdown of either Puma or Bax significantly reduced BV6/TMZ-mediated cell death (Figures $4 d$ and f). These results demonstrate that Puma and Bax contribute to BV6/TMZ-induced cell death.

\section{Discussion}

We previously reported NF- $k \mathrm{~B}$-dependent sensitization of glioblastoma cells to TMZ-induced apoptosis by the Smac mimetic BV6 as a novel approach to enhance the efficacy of conventional chemotherapy in glioblastoma. ${ }^{12}$ However, the proapoptotic NF-kB target genes mediating this synergistic interaction have so far remained elusive, as autocrine/ paracrine TNFa/TNF receptor 1 (TNFR1) signaling turned out to be largely dispensable. ${ }^{12}$ In the present study, we identify Smac mimetic-stimulated, NF- $\kappa$ B-dependent upregulation of IFN $\beta$ and IFN-mediated proapoptotic signaling as critical events that mediate BV6/TMZ-induced apoptosis (Supplementary Figure 5). This conclusion is based on the following lines of evidence:

First, treatment with BV6 alone or in combination with $\mathrm{TMZ}$ triggers transcriptional upregulation of IFN $\beta$ in an NF- $k \mathrm{~B}$ dependent manner, as this increase in IFN $\beta$ mRNA levels is blocked by $I_{k \mathrm{~B} a-S R}$-mediated inhibition of $\mathrm{NF}-\kappa \mathrm{B}$. In addition, gene expression profiling shows an NF- $k \mathrm{~B}$ dependent upregulation of ISGs upon BV6/TMZ treatment. Second, BV6-induced upregulation of IFN $\beta$ - and IFNmediated signaling are required for the induction of apoptosis, as genetic silencing of either IFN $\beta$ or its corresponding receptor IFNAR significantly reduces BV6/TMZ-induced apoptosis. The notion that BV6-stimulated upregulation of 

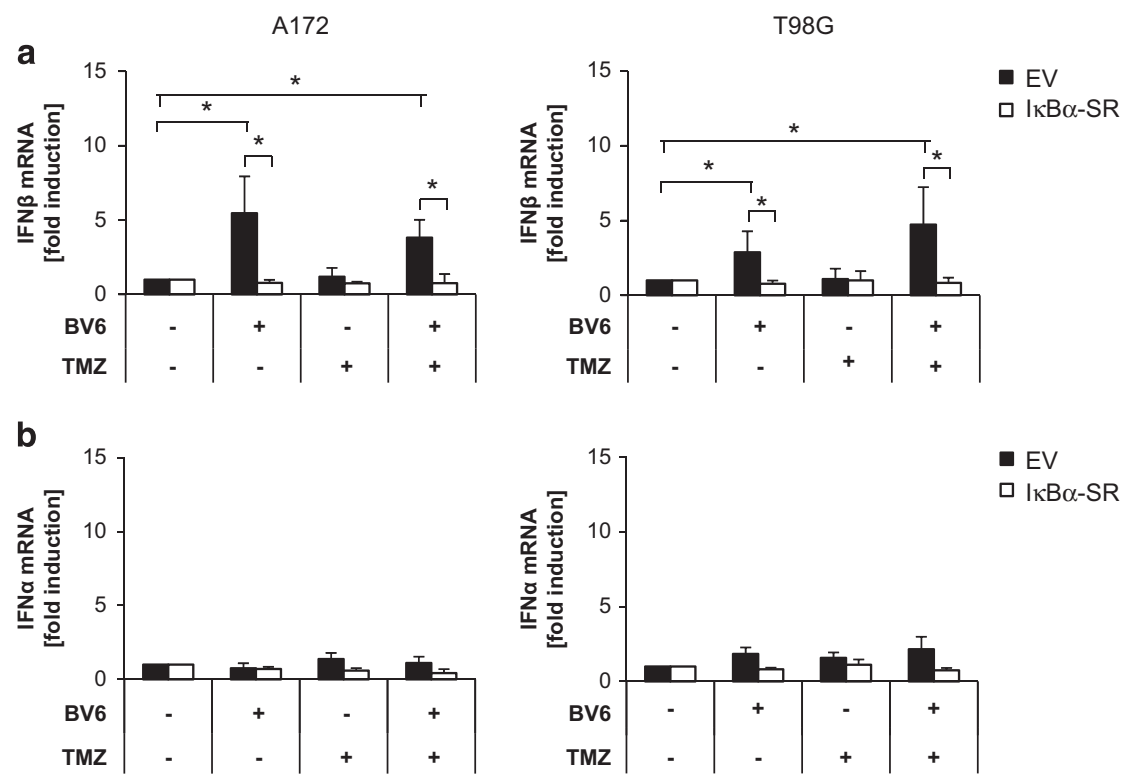

- EV

$\square \mathrm{I} \mathrm{KB} \alpha-\mathrm{SR}$
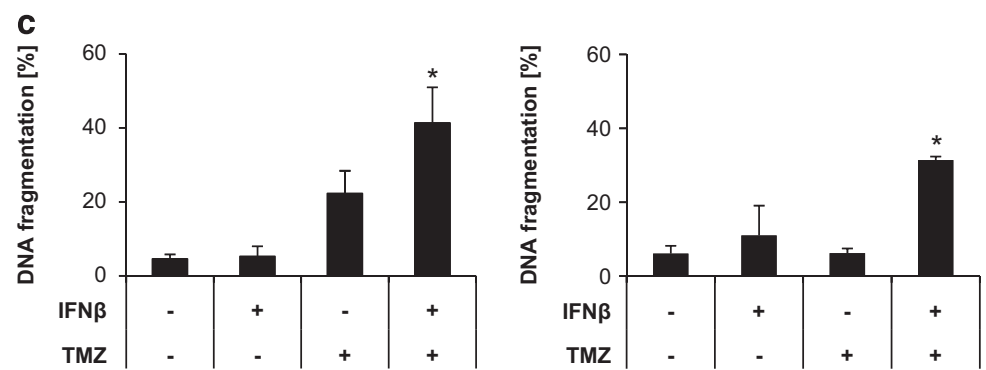

Figure 2 BV6-mediated upregulation of IFN $\beta$ sensitizes glioblastoma cells to TMZ-induced apoptosis. A172 cells (left) or T98G cells (right) stably expressing I $\kappa$ B $\alpha$-SR or EV were treated for $6 \mathrm{~h}$ with $100 \mu \mathrm{M} \mathrm{TMZ}$ and/or $2 \mu \mathrm{M}$ BV6 (A172) or $4 \mu \mathrm{M} \mathrm{BV6} \mathrm{(T98G)} \mathrm{or} \mathrm{dimethyl} \mathrm{sulfoxide} \mathrm{(DMSO).} \mathrm{IFN} \beta$ (a) or IFN $\alpha$ (b) mRNA levels were analyzed by qRT-PCR, normalized to $28 \mathrm{~S} \mathrm{rRNA}$ expression and fold increase in $\mathrm{mRNA}$ levels are shown. Mean values + S.D. of three to four independent experiments performed in duplicate are shown. ${ }^{*} P<0.05$; ${ }^{* *} P<0.01$. (c) A172 cells (left) or T98G cells (right) were treated for $120 \mathrm{~h}$ with $100 \mu \mathrm{M} \mathrm{TMZ}$ and/or $1 \mathrm{ng} / \mathrm{ml}$ IFN $\beta$ or DMSO. Apoptosis was determined by fluorescence-activated cell sorting (FACS) analysis of DNA fragmentation of PI-stained nuclei. Mean values + S.D. of three independent experiments performed in triplicate are shown; ${ }^{*} P<0.05 ;{ }^{* \star} P<0.01$

IFN $\beta$ promotes TMZ-induced apoptosis is further underscored by data showing that exogenous supply of IFN $\beta$ cooperates with TMZ to trigger apoptosis in glioblastoma cells. Third, we show that IFN $\beta$ and TMZ cooperate to upregulate the proapoptotic $\mathrm{Bcl}-2$ family proteins Puma and Bax, which both contribute to BV6/TMZ-induced apoptosis, as knockdown of Bax or Puma significantly rescues cells from BV6/TMZinduced apoptosis. Taken together, this identification of Smac mimetic-stimulated, NF- $k$ B-dependent upregulation of IFN $\beta$ and engagement of proapoptotic IFN signaling pathways provides novel insights into the molecular mechanisms that are responsible for Smac mimetic-mediated sensitization of glioblastoma cells to TMZ-induced cell death.

In the present study, we identify IFN $\beta$ as a key mediator of $\mathrm{BV} 6 / \mathrm{TMZ}$-induced cell death that is transcriptionally upregulated in an NF-kB-dependent manner upon treatment with the Smac mimetic BV6. Whether or not this increase in IFN $\beta$ is directly mediated via NF- $k B$ transcription factors ${ }^{20}$ or indirectly mediated via NF-KB-dependent upregulation or activation of transcription factors regulating IFN $\beta^{21}$ remains to be investigated in future studies. It is interesting to note that type I IFNs such as IFN $\beta$ or IFN $a$ have recently been reported to act in concert with TMZ to trigger cell death in glioblastoma cells, although the mechanisms responsible for this cooperative effect have so far remained elusive. ${ }^{16,17}$ In contrast to the critical role of IFN $\beta$ for BV6/TMZ-induced apoptosis that we discovered in the current study, we previously reported that TNF $a$, another prototypic NF- $\kappa$ B target gene, is largely dispensable for BV6/TMZ-induced apoptosis, as addition of the TNFa-blocking antibody Enbrel or TNFR1 knockdown failed to rescue apoptosis upon combination treatment. ${ }^{12}$

Although our study demonstrates for the first time that the Smac mimetic BV6 can transcriptionally induce IFN $\beta$ as an important mediator of Smac mimetic-conferred chemosensitization in glioblastoma cells, IFN signaling has been implicated in the past to foster cell death by Smac mimetics. For example, we recently reported that BV6 synergizes with IFNa to trigger apoptosis in acute myeloid leukemia cells. ${ }^{22}$ Of note, BV6 was found in the present study to transcriptionally upregulate IFN $\beta$, but not IFN $\alpha$ in glioblastoma cells, pointing to distinct roles of type I IFNs in this context. Moreover, we identified IFN regulatory factor 1 (IRF1) as a novel dual regulator of Smac mimetic BV6-induced apoptosis and 
a

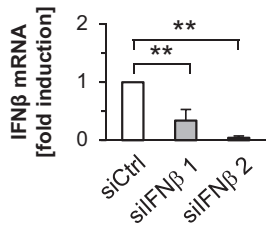

b

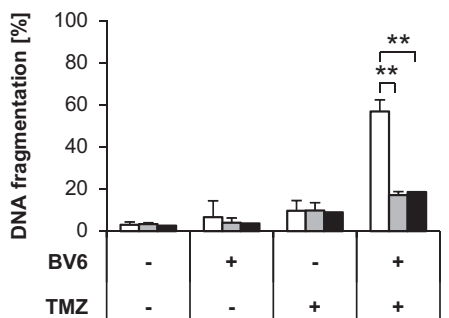

c

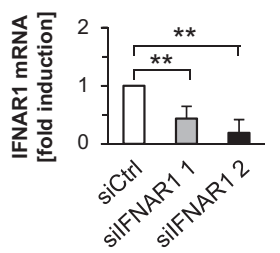

d
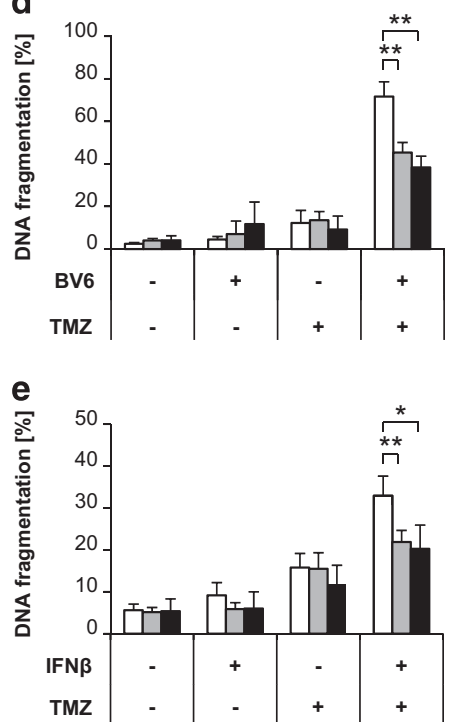
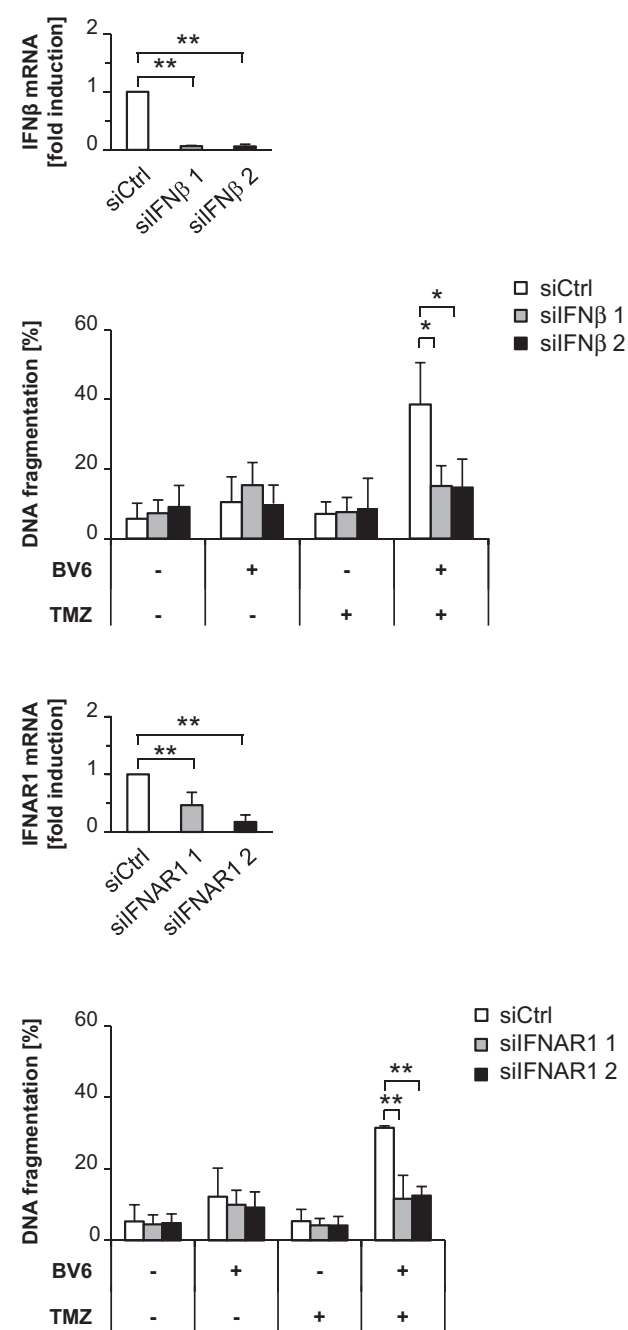

$\square$ siCtrl

SilFNAR1 1

- silFNAR1 2<smiles>C1CCCCC1</smiles>

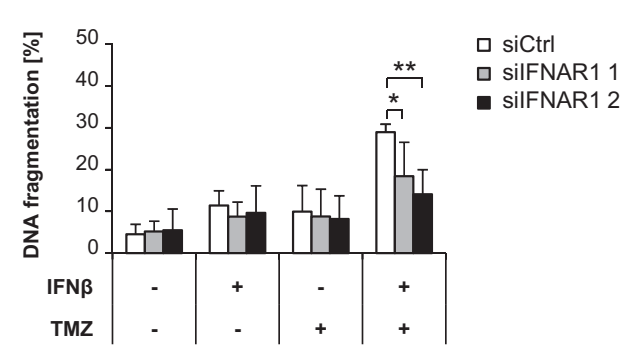

Figure 3 IFN $\beta$ is a crucial mediator of BV6/TMZ-induced cell death. (a) A172 cells (left) or T98G cells (right) were transiently transfected with small interfering RNA (siRNA) against IFN $\beta$. IFN $\beta$ mRNA levels were analyzed after $120 \mathrm{~h}$ by qRT-PCR, normalized to $28 \mathrm{~S}$ rRNA expression and fold increase in mRNA levels are shown. Mean values + S.D. of three independent experiments performed in duplicate are shown. ${ }^{*} P<0.05$; ${ }^{*} P<0.01$. (b) A172 cells (left) or T98G cells (right) were transiently transfected with siRNA against IFN $\beta$. Cells were treated for $120 \mathrm{~h}$ with $100 \mu \mathrm{M}$ TMZ and/or $2 \mu \mathrm{M}$ BV6 (A172) or $4 \mu \mathrm{M}$ BV6 (T98G) or dimethyl sulfoxide (DMSO). Apoptosis was determined by fluorescenceactivated cell sorting (FACS) analysis of DNA fragmentation of Pl-stained nuclei. Mean values + S.D. of three to four independent experiments performed in triplicate are shown; ${ }^{*} P<0.05 ;{ }^{*} P<0.01$. (c) A172 cells (left) or T98G cells (right) were transiently transfected with siRNA against IFNAR1. IFNAR1 mRNA levels were analyzed after $120 \mathrm{~h}$ by qRT$\mathrm{PCR}$, normalized to $28 \mathrm{~S}$ rRNA expression and fold increase in mRNA levels are shown. Mean values + S.D. of three to four independent experiments performed in duplicate are shown. ${ }^{*} P<0.05$; ${ }^{* *} P<0.01$. (d) A172 cells (left) or T98G cells (right) were transiently transfected with siRNA against IFNAR1. Cells were treated for $120 \mathrm{~h}$ with $100 \mu \mathrm{M}$ TMZ and/or $2 \mu \mathrm{M}$ BV6 (A172) or $4 \mu \mathrm{M} \mathrm{BV6} \mathrm{(T98G)} \mathrm{or} \mathrm{DMSO.} \mathrm{Apoptosis} \mathrm{was} \mathrm{determined} \mathrm{by} \mathrm{FACS} \mathrm{analysis} \mathrm{of} \mathrm{DNA} \mathrm{fragmentation} \mathrm{of} \mathrm{PI-stained} \mathrm{nuclei.} \mathrm{Mean} \mathrm{values} \mathrm{+} \mathrm{S.D.} \mathrm{of} \mathrm{three}$ independent experiments performed in triplicate are shown; ${ }^{*} P<0.05$; ${ }^{* *} P<0.01$. (e) A172 cells (left) or T98G cells (right) were transiently transfected with siRNA against IFNAR1. Cells were treated for $120 \mathrm{~h}$ with $100 \mu \mathrm{M}$ TMZ and/or $1 \mathrm{ng} / \mathrm{ml}$ IFN $\beta$ or DMSO. Apoptosis was determined by FACS analysis of DNA fragmentation of PI-stained nuclei. Mean values +S.D. of four independent experiments performed in triplicate are shown; ${ }^{*} P<0.05$; ${ }^{* \star} P<0.01$ 
proinflammatory cytokine secretion with impact on the immune response. ${ }^{23,24}$ Furthermore, Smac mimetics have been described to act in concert with innate immune stimuli such as oncolytic viruses and adjuvants, which stimulate a cytokine storm of TNFa, TNF-related apoptosis-inducing ligand and IFN $\beta$, to trigger cancer cell death. ${ }^{25}$
A172
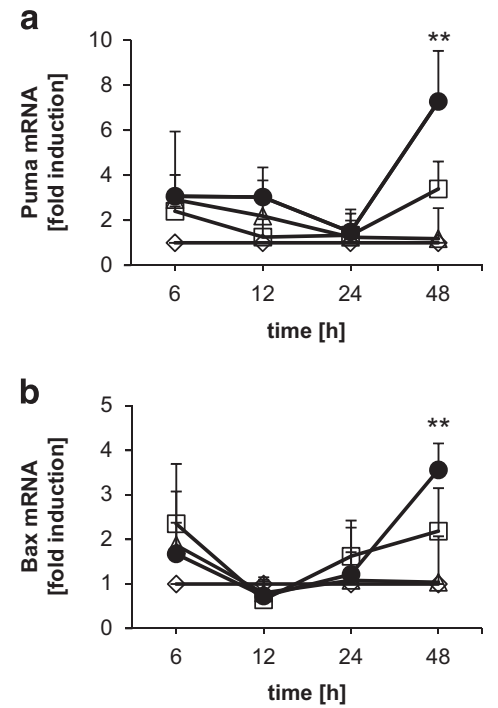

C
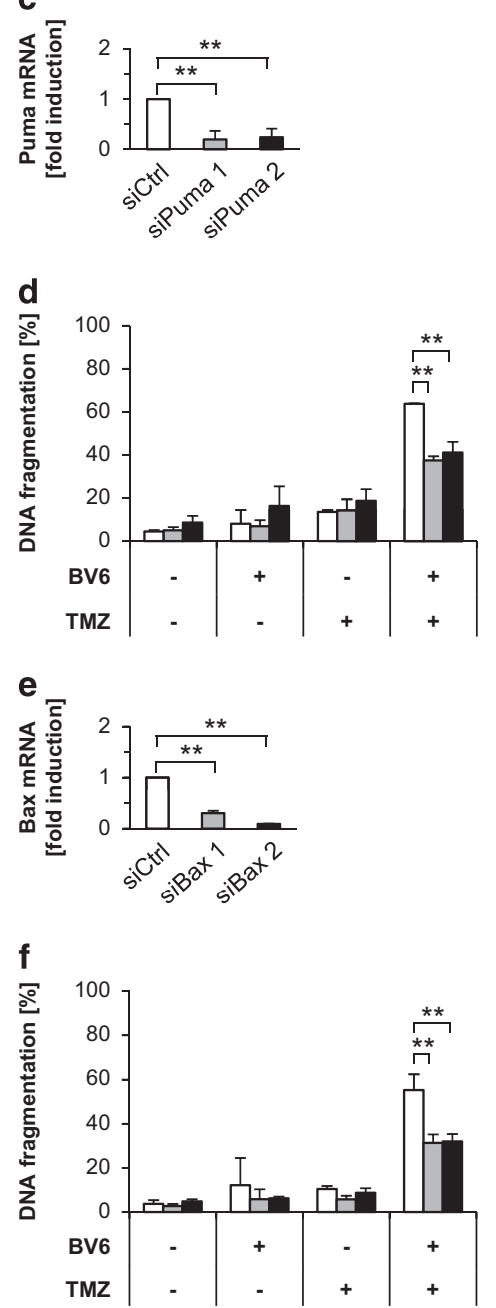

T98G
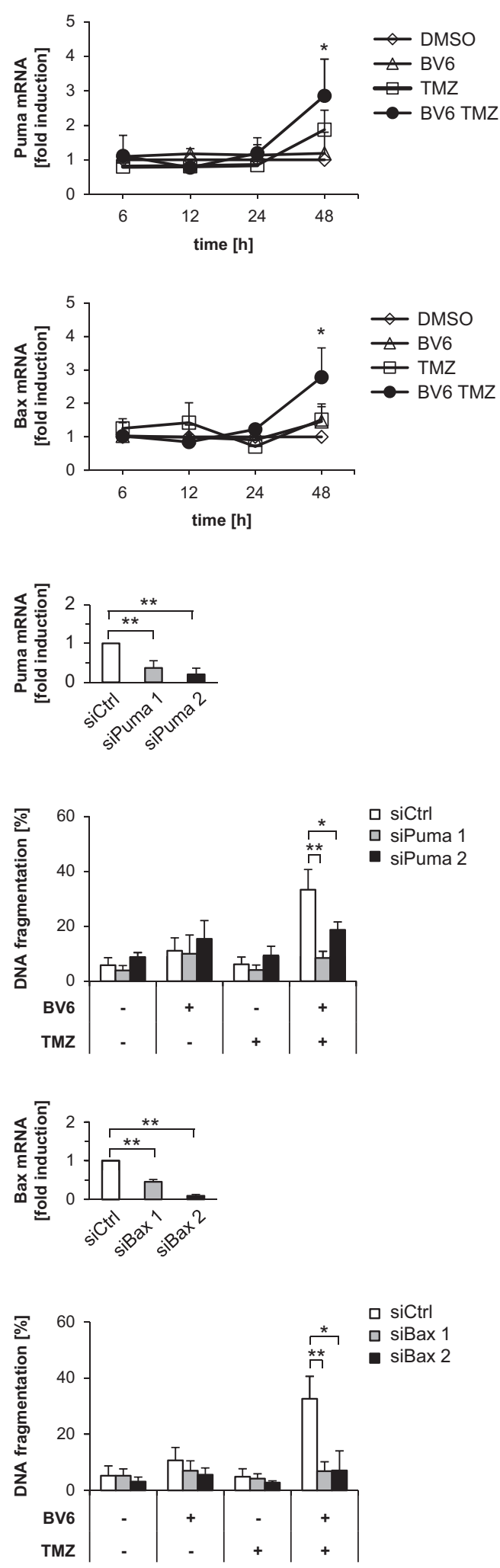
Induction of cell death by IFN $\beta$ has been described to involve ISGs. ${ }^{19}$ However, little is yet known about which ISGs mediate these apoptotic functions. Transcription factors regulated via IFNs such as IRF1 and IRF3 have been reported to promote upregulation or activation of Puma and Bax. ${ }^{26,27}$ Puma and Bax were also described as DNA damage-induced target genes that are upregulated by $\mathrm{TMZ}$ treatment. ${ }^{28}$ Consistently, we show in the present study that IFN $\beta$ and TMZ cooperate to upregulate Puma and Bax, which both contribute to BV6/TMZ-induced apoptosis, as genetic silencing of either Bax or Puma, two Bcl-2 family proteins known to promote mitochondrial apoptosis, rescues glioblastoma cells from cell death. In line with these findings, we previously reported that cotreatment with BV6/TMZ activates the mitochondrial pathway of apoptosis as demonstrated by the loss of mitochondrial membrane potential and cytochrome $C$ release. $^{12}$

Furthermore, context-specific settings have an impact on the regulation of signaling pathways and cellular functions by Smac mimetics, depending, for example, on additional cytotoxic stimuli and/or cell types. We demonstrated that Smac mimetics can exert non-apoptotic functions and can stimulate migration and invasion of glioblastoma cells via activation of NF- $\mathrm{KB}$ and TNFa/TNFR1 autocrine/paracrine signaling. ${ }^{23,24}$ In glioblastoma cancer stem-like cells, Smac mimetics at non-toxic concentrations can promote astrocytic differentiation by activating NF- $k \mathrm{~B}^{29}$

Smac mimetics are currently evaluated in early clinical trials. ${ }^{30}$ By identifying IFN $\beta$ as a novel Smac mimetic-induced and NF- $\mathrm{KB}$-mediated target gene that has an important role in mediating chemosensitization by Smac mimetic, our findings provide novel mechanistic insights into this combination regimen. Additionally, our present study emphasizes that Smac mimetics are effective sensitizers for TMZ-induced apoptosis in glioblastoma cells with implications for the development of experimental treatment approaches.

\footnotetext{
Materials and Methods

Cell culture and chemicals. The human glioblastoma cell lines A172 and T98G were obtained from the American Type Culture Collection (Manassas, VA, USA) and maintained in DMEM medium (Invitrogen, Karlsruhe, Germany) supplemented with $1 \%$ penicillin/streptomycin, $1 \%$ sodium pyruvate and $10 \%$ fetal calf serum (Invitrogen). For experiments, cells were seeded at $5 \times 10^{3} \mathrm{cells} / \mathrm{cm}^{2}$. Smac mimetic BV6, which neutralizes XIAP, CIAP1 and cIAP2, ${ }^{8}$ was kindly provided by Genentech Inc. (South San Francisco, CA, USA), TMZ was purchased from Sigma (Taufkirchen, Germany) and recombinant human IFN $\alpha$ and IFN $\beta$ from
}

Biochrom (Berlin, Germany). All chemicals were obtained from Sigma, unless indicated otherwise.

Determination of apoptosis. Apoptosis was assessed by flow cytometric analysis (FACSCanto II; BD Biosciences, Heidelberg, Germany) of DNA fragmentation of propidium iodide (PI)-stained nuclei as described previously. ${ }^{31}$

Western blotting. Western blot analysis was performed as described previously ${ }^{12}$ using the following antibodies: anti-IкB $\alpha$ (Cell Signaling, Beverly, MA, USA), anti-Bax (BD Biosciences), anti-Puma (Cell Signaling) and anti- $\beta$-actin (Sigma). Donkey anti-mouse IgG or donkey anti-rabbit IgG labeled with IRDye infrared dyes were used for fluorescence detection at $680-800 \mathrm{~nm}$ (LI-COR Biotechnology, Bad Homburg, Germany).

Whole-genome gene expression array and GSEA. Gene expression profiling was performed as described previously ${ }^{32}$ using Illumina Whole-Genome Expression Beadchips Human HT12v4 (Illumina, San Diego, CA, USA). Expression data were ranked according to fold upregulation comparing $A 172$ glioblastoma cells expressing EV with and without BV6/TMZ treatment. Expression data showing upregulation with and without BV6/TMZ treatment in A172 glioblastoma cells expressing $I_{\kappa} \mathrm{B} \alpha$-SR served as control to identify background expression of non-NF- $\kappa$ B-regulated genes. GSEA was performed using software provided by the Broad Institute website (http://www.broadinstitute. org/gsea/index.jsp). ${ }^{33}$

Transduction and siRNA transfection. Overexpression of the dominantnegative $\mid \kappa B \alpha-S R$ was performed by retroviral transduction as described previously. ${ }^{15}$ For transient knockdown by siRNA, cells were transfected with $20 \mathrm{nM}$ Silencer Select siRNA (Invitrogen) control siRNA (no. 4390844) or targeting siRNAs (s7188 and s7189 for IFN $\beta$, s782 and s784 for IFNAR1, s1888 and s1890 for Bax, s25840 and s25842 for Puma) using Neon Transfection System (Invitrogen) according to the manufacturer's instructions.

qRT-PCR analysis. Total RNA extraction and qRT-PCR analysis was performed as described previously ${ }^{32}$ using $7900 \mathrm{HT}$ Fast Real-Time PCR System (Applied Biosystems, Darmstadt, Germany). The following primers were used: $28 \mathrm{~S}$ (forward, 5'-TTGAAAATCCGGGGGAGAG-3'; reverse, 5'-ACATTGTTCCAACA TGCCAG-3'), IFNAR1 (forward, 5'-TCCAGTACATTGTATAAAGACCACAGT-3'; reverse, 5'-GTTCTGATTTTGGACACTGACTTC-3'), Puma (forward, 5'-GACCTC AACGCACAGTACGA-3'; reverse, 5'-GAGATTGTACAGGACCCTCCA-3'), Bax (forward, 5'-AGCAAACTGGTGCTCAAGG-3'; reverse, 5'-TCTTGGATCCAGCC CAAC-3'), Bak (forward, 5'-CCTGCCCTCTGCTTCTGA-3'; reverse, 5'-CTGCTGA TGGCGGTAAAAA-3'), Noxa (forward, 5'-GGAGATGCCTGGGAAGAAG-3'; reverse, 5'-CCTGAGTTGAGTAGCACACTCG-3'), Bid (forward, 5'-TGCAGCTCA GGAACACCA-3'; reverse, 5'-TCTCCATGTCTCTAGGGTAGGC-3'), Bim (forward, 5'-CATCGCGGTATTCGGTTC-3'; reverse, 5'-GCTTTGCCATTTGGTCTTTTT-3'), Bmf (forward, 5'-GAGACTCTCTCCTGGAGTCACC-3'; reverse, 5'-CTGGTTGGA ACACATCATCCT- $3^{\prime}$ ). Melting curves were plotted to verify the specificity of the amplified products. IFN $\alpha$ and IFN $\beta$ mRNA levels were assessed by TaqMan Gene Expression Assay (Life Technologies, Darmstadt, Germany; IFN $\alpha$ Hs01077958_s1, IFN $\beta$ Hs00855471_g1) according to the manufacturer's protocol. The relative expression of the target gene transcript and reference gene transcript was calculated as $\Delta \Delta \mathrm{C}_{\mathrm{t}}$.

Figure 4 BV6/TMZ-induced apoptosis is mediated by the upregulation of Puma and Bax. A172 cells (left) or T98G cells (right) were treated for indicated times with $100 \mu \mathrm{M}$ TMZ and/or $2 \mu \mathrm{M}$ BV6 (A172) or $4 \mu \mathrm{M}$ BV6 (T98G) or dimethyl sulfoxide (DMSO). Puma (a) or Bax (b) mRNA levels were analyzed by qRT-PCR, normalized to 28S rRNA expression and fold increase in mRNA levels are shown. Mean values + S.D. of three independent experiments performed in duplicate are shown. ${ }^{*} P<0.05 ;{ }^{* \star} P<0.01$ compared with DMSO control. (c) A172 cells (left) or T98G cells (right) were transiently transfected with small interfering RNA (siRNA) against Puma. Puma mRNA levels were analyzed after $120 \mathrm{~h}$ by qRT-PCR, normalized to $28 \mathrm{~S}$ rRNA expression and fold increase in mRNA levels are shown. Mean values + S.D. of three independent experiments performed in duplicate are shown. ${ }^{*} P<0.05$; ${ }^{* *} P<0.01$ compared with all other settings, if not indicated otherwise. (d) A172 cells (left) or T98G cells (right) were transiently transfected with siRNA against Puma. Cells were treated for $120 \mathrm{~h}$ with $100 \mu \mathrm{M} \mathrm{TMZ}$ and/or $2 \mu \mathrm{M}$ BV6 (A172) or $4 \mu \mathrm{M}$ BV6 (T98G) or DMSO. Apoptosis was determined by fluorescenceactivated cell sorting (FACS) analysis of DNA fragmentation of PI-stained nuclei. Mean values + S.D. of three to four independent experiments performed in triplicate are shown; ${ }^{*} P<0.05$; ${ }^{*} P<0.01$. (e) A172 cells (left) or T98G cells (right) were transiently transfected with siRNA against Bax. Bax mRNA levels were analyzed after $120 \mathrm{~h}$ by $q R T-P C R$, normalized to $28 \mathrm{~S}$ rRNA expression and fold increase in mRNA levels are shown. Mean values + S.D. of three independent experiments performed in duplicate are shown. ${ }^{*} P<0.05 ;{ }^{*} P<0.01$. (f) A172 cells (left) or T98G cells (right) were transiently transfected with siRNA against Bax. Cells were treated for $120 \mathrm{~h}$ with $100 \mu \mathrm{M} \mathrm{TMZ}$ and/or $2 \mu \mathrm{M}$ BV6 (A172) or $4 \mu \mathrm{M}$ BV6 (T98G) or DMSO. Apoptosis was determined by FACS analysis of DNA fragmentation of Pl-stained nuclei. Mean values + S.D. of three independent experiments performed in triplicate are shown; ${ }^{*} P<0.05 ;{ }^{* *} P<0.01$ 
Statistical analysis. Statistical significance was assessed by Student's t-test (two-tailed distribution, two-sample unequal variance).

\section{Conflict of Interest}

The authors declare no conflict of interest.

Acknowledgements. We thank D Vucic (Genentech Inc.) for providing BV6, the microarray unit of the DKFZ Genomics and Proteomics Core Facility (Heidelberg, Germany) for providing the Illumina Whole-Genome Expression Beadchips and related services, Ronald Sauter for expert technical assistance and $C$ Hugenberg for expert secretarial assistance. This work has been partially supported by grants from the Deutsche Forschungsgemeinschaft, the BMBF and IUAPVII (to SF).

1. Stupp R, Hegi ME, Mason WP, van den Bent MJ, Taphoorn MJ, Janzer RC et al. Effects of radiotherapy with concomitant and adjuvant temozolomide versus radiotherapy alone on survival in glioblastoma in a randomised phase III study: 5-year analysis of the EORTCNCIC trial. Lancet Oncol 2009; 10: 459-466.

2. Van Meir EG, Hadjipanayis CG, Norden AD, Shu HK, Wen PY, Olson JJ. Exciting new advances in neuro-oncology: the avenue to a cure for malignant glioma. CA Cancer $J$ Clin 2010; 60: 166-193.

3. Hanahan D, Weinberg RA. Hallmarks of cancer: the next generation. Cell 2011; 144: 646-674.

4. Fulda S. Tumor resistance to apoptosis. Int J Cancer 2009; 124: 511-515.

5. Fulda S, Debatin KM. Extrinsic versus intrinsic apoptosis pathways in anticancer chemotherapy. Oncogene 2006; 25: 4798-4811.

6. Fulda S, Galluzzi L, Kroemer G. Targeting mitochondria for cancer therapy. Nat Rev Drug Discov 2010; 9: 447-464.

7. Fulda S, Vucic D. Targeting IAP proteins for therapeutic intervention in cancer. Nat Rev Drug Discov 2012; 11: 109-124.

8. Varfolomeev E, Blankenship JW, Wayson SM, Fedorova AV, Kayagaki N, Garg P et al. IAP antagonists induce autoubiquitination of c-IAPs, NF-kappaB activation, and TNFalphadependent apoptosis. Cell 2007; 131: 669-681.

9. Vince JE, Wong WW, Khan N, Feltham R, Chau D, Ahmed AU et al. IAP antagonists target clAP1 to induce TNFalpha-dependent apoptosis. Cell 2007; 131: 682-693.

10. Vellanki SH, Grabrucker A, Liebau S, Proepper C, Eramo A, Braun V et al. Small-molecule XIAP inhibitors enhance gamma-irradiation-induced apoptosis in glioblastoma. Neoplasia 2009; 11: 743-752.

11. Berger R, Jennewein C, Marschall V, Karl S, Cristofanon S, Wagner L et al. NF-\{kappa\}B is required for Smac mimetic-mediated sensitization of glioblastoma cells for \{gamma\}irradiation-induced apoptosis. Mol Cancer Ther 2011; 10: 1867-1875.

12. Wagner L, Marschall V, Karl S, Cristofanon S, Zobel K, Deshayes K et al. Smac mimetic sensitizes glioblastoma cells to temozolomide-induced apoptosis in a RIP1- and NF-kappaBdependent manner. Oncogene 2013; 32: 988-997.

13. Petersen SL, Wang L, Yalcin-Chin A, Li L, Peyton M, Minna J et al. Autocrine TNFalpha signaling renders human cancer cells susceptible to Smac-mimetic-induced apoptosis. Cancer Cell 2007; 12: 445-456.

14. Probst BL, Liu L, Ramesh V, Li L, Sun H, Minna JD et al. Smac mimetics increase cancer cell response to chemotherapeutics in a TNF-alpha-dependent manner. Cell Death Differ 2010; 17: $1645-1654$.

15. Karl S, Pritschow $Y$, Volcic M, Hacker S, Baumann B, Wiesmuller $L$ et al. Identification of a novel pro-apopotic function of NF-kappaB in the DNA damage response. J Cell Mol Med 2009; 13: 4239-4256.
16. Park JA, Joe YA, Kim TG, Hong YK. Potentiation of antiglioma effect with combined temozolomide and interferon-beta. Oncol Rep 2006; 16: 1253-1260.

17. Yoshino A, Tashiro S, Ogino A, Yachi K, Ohta T, Fukushima T et al. Gene expression profiles predicting the response to IFN-beta and a combination of temozolomide and IFN-beta in malignant gliomas. Int J Oncol 2011; 39: 529-542.

18. Stark GR, Darnell JE Jr. The JAK-STAT pathway at twenty. Immunity 2012; 36: 503-514.

19. Kotredes KP, Gamero AM. Interferons as inducers of apoptosis in malignant cells. $J$ Interferon Cytokine Res 2013; 33: 162-170.

20. Lenardo MJ, Fan CM, Maniatis T, Baltimore D. The involvement of NF-kappa B in betainterferon gene regulation reveals its role as widely inducible mediator of signal transduction. Cell 1989; 57: 287-294.

21. Ivashkiv LB, Donlin LT. Regulation of type I interferon responses. Nat Rev Immunol 2014; 14: 36-49.

22. Bake V, Roesler S, Eckhardt I, Belz K, Fulda S. Synergistic interaction of Smac mimetic and IFNalpha to trigger apoptosis in acute myeloid leukemia cells. Cancer Lett 2014; 355: 224-231.

23. Tchoghandjian A, Jennewein C, Eckhardt I, Rajalingam K, Fulda S. Identification of noncanonical NF-kappaB signaling as a critical mediator of Smac mimetic-stimulated migration and invasion of glioblastoma cells. Cell Death Dis 2013; 4: e564.

24. Eckhardt I, Weigert A, Fulda S. Identification of IRF1 as critical dual regulator of Smac mimeticinduced apoptosis and inflammatory cytokine response. Cell Death Dis 2014; 5: e1562.

25. Beug ST, Tang VA, LaCasse EC, Cheung HH, Beauregard CE, Brun J et al. Smac mimetics and innate immune stimuli synergize to promote tumor death. Nat Biotechnol 2014; 32: $182-190$.

26. Chattopadhyay S, Marques JT, Yamashita M, Peters KL, Smith K, Desai et al. Viral apoptosis is induced by IRF-3-mediated activation of Bax. EMBO J 2010; 29: 1762-1773.

27. Gao J, Senthil M, Ren B, Yan J, Xing Q, Yu J et al. IRF-1 transcriptionally upregulates PUMA, which mediates the mitochondrial apoptotic pathway in IRF-1-induced apoptosis in cancer cells. Cell Death Differ 2010; 17: 699-709.

28. Borges HL, Linden R, Wang JY. DNA damage-induced cell death: lessons from the central nervous system. Cell Res 2008; 18: 17-26.

29. Tchoghandjian A, Jennewein C, Eckhardt I, Momma S, Figarella-Branger D, Fulda S. Smac mimetic promotes glioblastoma cancer stem-like cell differentiation by activating NF-kappaB. Cell Death Differ 2014; 21: 735-747.

30. Fulda S. Molecular pathways: targeting inhibitor of apoptosis proteins in cancer - from molecular mechanism to therapeutic application. Clin Cancer Res 2014; 20: 289-295.

31. Fulda S, Sieverts H, Friesen C, Herr I, Debatin KM. The CD95 (APO-1/Fas) system mediates drug-induced apoptosis in neuroblastoma cells. Cancer Res 1997; 57: 3823-3829.

32. Eckhardt I, Roesler S, Fulda S. Identification of DR5 as a critical, NF-kappaB-regulated mediator of Smac-induced apoptosis. Cell Death Dis 2013; 4: e936.

33. Subramanian A, Tamayo P, Mootha VK, Mukherjee S, Ebert BL, Gillette MA et al. Gene set enrichment analysis: a knowledge-based approach for interpreting genome-wide expression profiles. Proc Natl Acad Sci USA 2005; 102: 15545-15550.

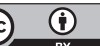

Cell Death and Disease is an open-access journal published by Nature Publishing Group. This work is licensed under a Creative Commons Attribution 4.0 International License. The images or other third party material in this article are included in the article's Creative Commons license, unless indicated otherwise in the credit line; if the material is not included under the Creative Commons license, users will need to obtain permission from the license holder to reproduce the material. To view a copy of this license, visit http://creativecommons.org/licenses/by/4.0/ 